\title{
The impact of the COVID-19 pandemic on self-harm and
}

\section{suicidal behaviour: protocol for a living systematic review}

\section{[version 1; peer review: 1 approved, 1 approved with}

\section{reservations]}

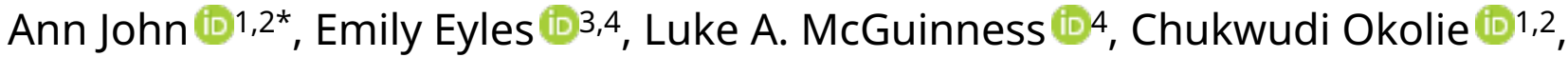 \\ Babatunde K. Olorisade (D) 4, Lena Schmidt (D) 4, Roger T. Webb5,6, Ella Arensman7, \\ Keith Hawton (id) 8,9, Nav Kapur5,6,10, Paul Moran4,11, Rory C. O'Connor ${ }^{12}$, \\ Siobhan O'Neill13, David Gunnell4,11*, Julian P.T. Higgins 3,4,11*
}

\footnotetext{
${ }^{1}$ Population Psychiatry, Suicide and Informatics, Swansea University, Swansea, UK

2Public Health Wales NHS Trust, Swansea, UK

${ }^{3}$ National Institute for Health Research Applied Research Collaboration West (NIHR ARC West) at University Hospitals Bristol NHS Foundation Trust, Bristol, UK

${ }^{4}$ Population Health Sciences, University of Bristol, Bristol, UK

${ }^{5}$ Division of Psychology and Mental Health, University of Manchester, Manchester, UK

${ }^{6}$ NIHR Greater Manchester Patient Safety Translational Research Centre, Manchester, UK

${ }^{7}$ School of Public Health and National Suicide Research Foundation, University College Cork, Cork, Ireland

8 University Department of Psychiatry, Centre for Suicide Research, University of Oxford, Oxford, UK

${ }^{9}$ Oxford NHS Foundation Trust, Oxford, UK

${ }^{10}$ Greater Manchester Mental Health NHS Foundation Trust, Manchester, UK

${ }^{11}$ National Institute for Health Research Biomedical Research Centre at the University Hospitals Bristol NHS Foundation Trust and the University of Bristol, Bristol, UK

${ }^{12}$ Institute of Health \& Wellbeing, University of Glasgow, Glasgow, UK

${ }^{13}$ School of Psychology, University of Ulster, Belfast, UK

${ }^{\star}$ Equal contributors
}

V1 First published: 25 Jun 2020, 9:644
https://doi.org/10.12688/f1000research.24274.1
Latest published: 25 Jun 2020, 9:644

https://doi.org/10.12688/f1000research.24274.1

\section{Abstract}

Background: The COVID-19 pandemic has caused widespread morbidity and mortality as well as disruption to people's lives and livelihoods around the world; this has occurred as a result of both infection with the virus itself and the health protection measures taken to curb its spread. There are concerns that rates of suicide, suicidal behaviours and self-harm may rise during and in the aftermath of the pandemic. Given the likely rapidly expanding research evidence base on the pandemic's impact on rates of suicide, suicidal behaviours and self-harm and emerging evidence about how best to mitigate such effects, it is important that the best available

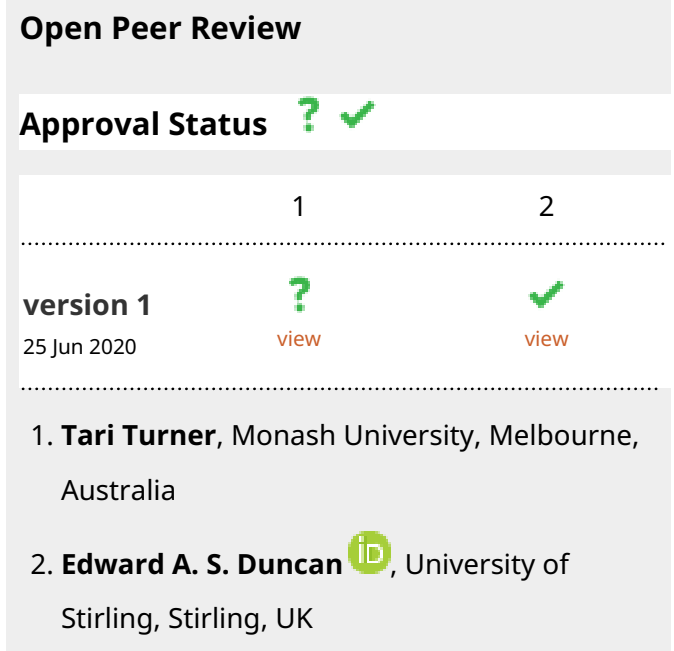


knowledge is made readily available to policymakers, public health specialists and clinicians as soon as is possible. To facilitate this, we plan to undertake a living systematic review focusing on suicide prevention in relation to COVID-19.

Method: Regular automated searches will feed into a web-based screening system which will also host the data extraction form for included articles. Our eligibility criteria are wide and include aspects of incidence and prevalence of suicidal behaviour, effects of exposures and effects of interventions in relation to the COVID-19 pandemic, with minimal restrictions on the types of study design to be included. The outcomes assessed will be death by suicide; self-harm or attempted suicide (including hospital attendance and/or admission for these reasons); and suicidal thoughts/ideation. There will be no restriction on study type, except for single case reports. There will be no restriction on language of publication. The review will be updated at three-monthly intervals if a sufficient volume of new evidence justifies doing so.

Conclusions: Our living review will provide a regular synthesis of the most up-to-date research evidence to guide public health and clinical policy to mitigate the impact of COVID-19 on suicide.

Protocol registration: PROSPERO CRD42020183326 01/05/2020

Keywords

COVID-19, Living systematic review, Suicide, Attempted suicide, Selfharm, Suicidal thoughts

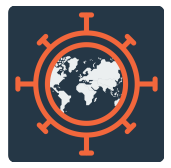

This article is included in the Emerging Diseases and Outbreaks gateway.

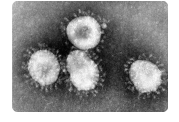

This article is included in the Coronavirus collection.

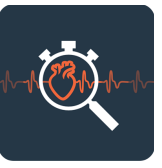

This article is included in the Living Evidence collection.
Any reports and responses or comments on the article can be found at the end of the article. 


\section{Corresponding author: Ann John (a.john@swansea.ac.uk)}

Author roles: John A: Conceptualization, Investigation, Methodology, Project Administration, Supervision, Writing - Original Draft Preparation, Writing - Review \& Editing; Eyles E: Data Curation, Investigation, Writing - Review \& Editing; McGuinness LA: Data Curation, Methodology, Resources, Software, Writing - Review \& Editing; Okolie C: Data Curation, Investigation, Writing - Original Draft Preparation; Olorisade BK: Methodology, Writing - Review \& Editing; Schmidt L: Data Curation, Methodology, Software, Visualization, Writing - Original Draft Preparation, Writing - Review \& Editing; Webb RT: Investigation, Writing - Review \& Editing; Arensman E: Writing - Review \& Editing; Hawton K: Writing - Review \& Editing; Kapur N: Writing - Review \& Editing; Moran P: Writing - Review \& Editing; O'Connor RC: Writing - Review \& Editing; O'Neill S: Writing - Review \& Editing; Gunnell D: Conceptualization, Investigation, Project Administration, Supervision, Writing - Original Draft Preparation, Writing - Review \& Editing; Higgins JPT: Conceptualization, Methodology, Project Administration, Supervision, Writing - Original Draft Preparation, Writing - Review \& Editing

Competing interests: DG: member of the Department of Health and Social Care (England) National Suicide Prevention Strategy Advisory Group. DG has grants from the National Institute for Health Research (NIHR) outside the submitted work and is a member of Samaritans Policy and Research Committee and Movember's Global Advisory Committee. AJ: chair of the National Advisory Group on Suicide and Self-harm Prevention to Welsh Government and is national lead on suicide prevention for Public Health Wales. KH: member of the Department of Health and Social Care (England) National Suicide Prevention Strategy Advisory Group. NK is a member of the Department of Health and Social Care (England) National Suicide Prevention Strategy Advisory Group and sits on committees for the National Institute for Health and Care Excellence to develop clinical guidelines for depression and self-harm. He reports grants outside the submitted work from NIHR, DHSC, and the Health Care Quality Improvement Partnership.

Grant information: This work was supported by Swansea University and the University of Bristol. DG, BKO, JPTH are supported by the NIHR Bristol Biomedical Research Centre [IS-BRC-1215-20011]. JPTH and EE are suported by the NIHR Applied Research Collaboration West. LAMcG is by the NIHR through a NIHR Doctoral Research Fellowship [DRF-2018-11-ST2-048]. LS is supported by the NIHR through a NIHR Systematic Reviews Fellowship [RM-SR-2017-09-028]. AJ and CO are supported by the Swansea University Cochrane Satellite for Suicide and Self-Harm Prevention. NK and RW are supported by the NIHR Greater Manchester Patient Safety Translational Research Centre.

The funders had no role in study design, data collection and analysis, decision to publish, or preparation of the manuscript.

Copyright: $(2020$ John A et al. This is an open access article distributed under the terms of the Creative Commons Attribution License, which permits unrestricted use, distribution, and reproduction in any medium, provided the original work is properly cited.

How to cite this article: John A, Eyles E, McGuinness LA et al. The impact of the COVID-19 pandemic on self-harm and suicidal behaviour: protocol for a living systematic review [version 1; peer review: 1 approved, 1 approved with reservations] F1000Research 2020, 9:644 https://doi.org/10.12688/f1000research.24274.1

First published: 25 Jun 2020, 9:644 https://doi.org/10.12688/f1000research.24274.1 


\section{Introduction}

The COVID-19 pandemic is causing widespread disruption and loss of life globally. By mid-May 2020 over 4.4 million people had been infected and 300,000 had died [https://www. worldometers.info/coronavirus/]. There are concerns about the impact of the pandemic on population mental health (Holmes et al., 2020). These stem from the impact of the virus itself on the people infected, as well as front line workers caring for them (Kisely et al., 2020), and from the public health measures to minimise spread of the virus - in particular physical distancing, leading to social isolation, disruption of work, businesses and education. Physical distancing measures have resulted in substantial rises in unemployment, falls in GDP and concerns that many nations will enter a period of deep economic recession.

There are concerns that suicide and self-harm rates may rise during and in the aftermath of the pandemic (Gunnell et al., 2020; Reger et al., 2020). Time-series modelling indicated that the 1918-20 Spanish Flu Pandemic, which caused well over 20 million deaths worldwide, led to a modest rise in the national suicide rate in the USA (Johnson \& Mueller, 2002; Wasserman, 1992). Likewise, there is evidence that suicide rates increased briefly amongst people aged over 65 in Hong Kong during the 2003 SARS epidemic, predominantly amongst those with more severe physical illness and physical dependency (Cheung et al., 2008).

The current context is, however, very different from previous epidemics and pandemics. The 2003 SARS epidemic was restricted to relatively few countries. Furthermore, in the time since the 1918-20 influenza pandemic, global and national health systems have improved, international travel and the speed of communication of information (and disinformation) have increased, antibiotics are available to treat secondary infection, and national economies have become more inter-dependent. The availability of the internet and technology for communication has made it easier for people to communicate and engage in home working and home schooling. However, there are huge inequalities in relation to access to technology and ability to stay safe and continue to work, within and between countries. Public health policies and responses, and the degree of access to technology to facilitate online clinical assessments and treatments differ greatly between countries.

Key concerns in relation to suicide prevention include: uncertainty regarding how best to assess and support people with suicidal thoughts and behaviours, whilst maintaining physical distancing; people who have attempted suicide may not attend hospitals because they are worried about contracting COVID-19 or being a burden on the healthcare system at this time; the uncertain impact on mental health and risk of suicide in the general population of physical distancing; an economic recession may adversely impact on suicide rates (Chang et al., 2013; Stuckler et al., 2009). There may be an increase in prevalence of suicide risk factors such as mental illness, domestic violence, alcohol misuse and bereavement (Turecki et al., 2019); the insensitive or irresponsible media reporting of suicide deaths associated with COVID-19 may be harmful; and in some countries access to highly lethal suicide methods such as firearms and pesticides may rise (Gunnell et al., 2020).
In the context of the COVID-19 pandemic there is likely to be a rapidly expanding research evidence base on its impact on suicide rates, and how best to mitigate such effects. It is therefore important that the best available knowledge is made rapidly available to policymakers, public health specialists and clinicians. To facilitate this, we plan to conduct a living systematic review focusing on suicide prevention in relation to COVID-19. Living systematic reviews are high-quality, up-todate online summaries of research that are regularly updated, using efficient, often semi-automated, systems of production (Elliott et al., 2014).

Aim

The overarching aim of the review is to identify and appraise any newly published evidence from around the world that assesses the impact of the COVID-19 pandemic on suicide deaths, suicidal behaviours, self-harm and suicidal thoughts, or that assesses the effectiveness of strategies to reduce the risk of suicide deaths, suicidal behaviours, self-harm and suicidal thoughts, resulting from the COVID-19 pandemic.

\section{Methods}

We will undertake a living systematic review (Figure 1) following published guidance for such reviews and for how expediated 'living' recommendations should be formed where relevant (Akl et al., 2017; Elliott et al., 2017). The review was prospectively registered (PROSPERO ID CRD42020183326; registered on 1 May 2020). This protocol is reported in line with the Preferred Reporting Items for Systematic Review and Meta-Analysis Protocols guideline (Moher et al., 2015).

\section{Eligibility criteria}

Study participants may be adults or children of any ethnicities living in any country. Outcomes of interest are:

1. Deaths by suicide

2. Self-harm (intentional self-injury or self-poisoning regardless of motivation and intent) or attempted suicide (including hospital attendance and/or admission for these reasons)

\section{Suicidal thoughts/ideation}

Studies must address one of the following research questions:

(i) What is the prevalence/incidence?

- Prevalence/incidence of each outcome during pandemic (including modelling studies to predict incidence / prevalence)

(ii) What is the comparative prevalence/incidence?

- Prevalence/incidence of each outcome during pandemic vs not during pandemic

(iii) What are the effects of interventions?

- Effects of public health measures to combat COVID-19 (including physical distancing, school closures, interventions to address loss of income, interventions to tackle domestic violence) on each outcome 


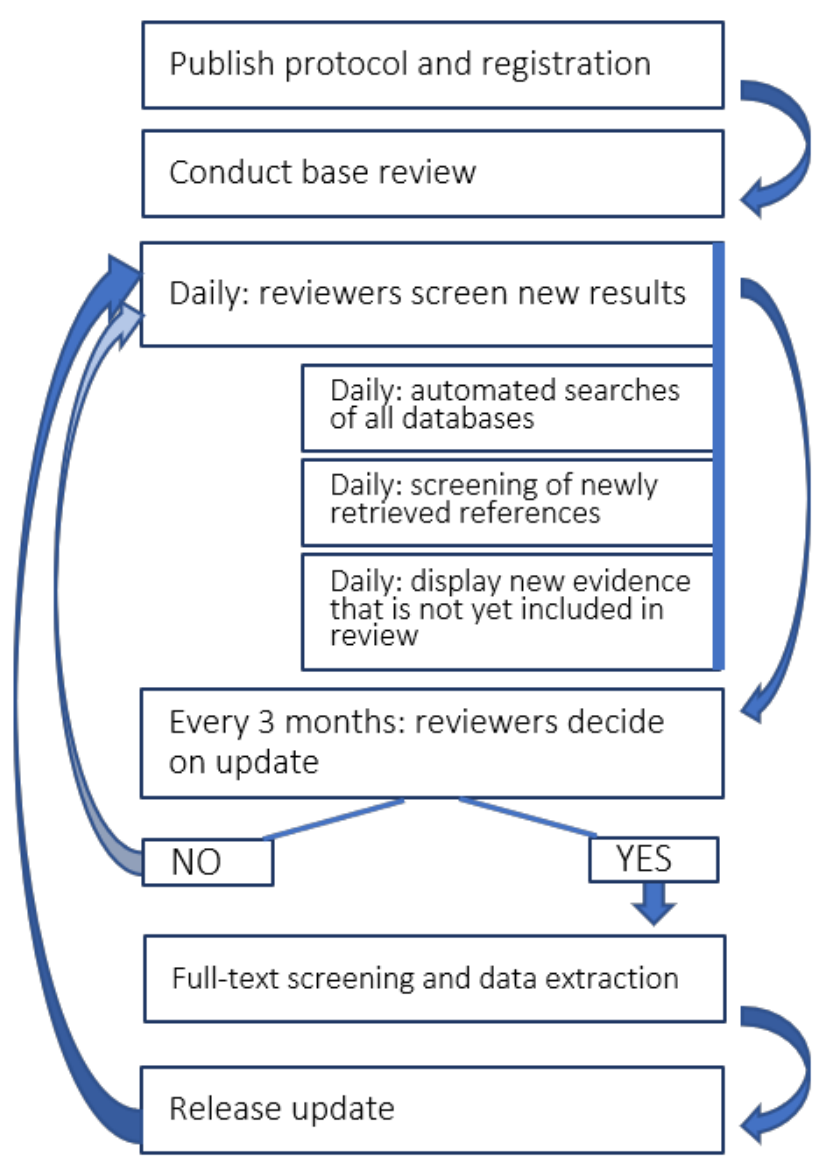

Figure 1. Workflow for updating the living review. The process will be supported using automation technology and in three-month intervals reviewers will decide on extracting new data and updating the review.

- Effects of changed and new approaches to clinical management of (perceived) elevated risk of self-harm or suicide risk on each outcome (any type of intervention is relevant)

(iv) What are the effects of other exposures?

- Impact of media portrayal of each outcome and misinformation attributed to the pandemic on each outcome

- Impact of bereavement from COVID-19 on each outcome

- Impact of any COVID-19 related behaviour changes (domestic violence, alcohol, cyberbullying) on each outcome

- Impact of COVID-19-related workload on crisis lines on each outcome

- Impact of infection with COVID-19 (self or family member) on each outcome

- Impact of increased availability of analgesics, firearms and pesticides on each outcome (method-specific and overall suicide rates)
- Impact of COVID-19 related socio-economic exposures (recession/depression: unemployment, debt, deprivation at the person-, family- or small-area level) on each outcome

- Impact on health and social care professionals: the stigma of working with COVID-19 patients or the (perceived) risk of infection/being a 'carrier', as well as workrelated stress on each outcome

- Impact of changes in/reduced intensity of treatment for patients with mental health conditions, in particular those with severe psychiatric disorders.

- Impact of any other relevant exposure on our outcomes of interest.

Qualitative research. We will include any qualitative research addressing perceptions or experiences around each outcome in relation to the COVID-19 pandemic (e.g. stigma of infection, isolation measures, complicated bereavement, media reporting, experience of remote methods of self-harm / suicide risk assessment or provision of treatment; experience of seeking help for 
individuals in suicidal crisis); narratives provided for precipitating factors for each outcome.

No restrictions will be placed on the types of study design eligible for inclusion, with the exception of single case reports. Pre-prints will be assessed at the time of publication. There will be no restriction on language of publication. We will draw on a combination of internet-based translation systems and network of colleagues to translate evidence in a language other than English.

\section{Identification of eligible studies}

We will search the following electronic databases: PubMed; Scopus; medRxiv, bioRxiv; the COVID-19 Open Research Dataset (CORD-19) by Semantic Scholar and the Allen Institute for AI, includes relevant records from Microsoft Academic, Elsevier, arXiv and PMC); and the WHO COVID-19 database. A sample search strategy (for PubMed) appears in Box 1. We have developed a workflow that automates daily searches of these databases, and the code supporting this process can be found at https://github.com/mcguinlu/COVID_suicide_living (McGuinness \& Schmidt, 2020)). Searches are conducted daily via PubMed and Scopus application program interface and the bioRxiv and medRxiv RSS feeds. Conversion scripts for the daily updated WHO and the weekly updated CORD-19 corpus are used to collect information from the remaining sources. The software includes a systematic search function based on regular expressions to search results retrieved from the WHO, CORD-19 and preprint repositories (search strategy available as extended data (John et al., 2020)). We will investigate the use of other databases to capture papers made available prior to peer review and assess eligibility and review internally.

\section{Box 1. Search terms for PubMed \\ ((selfharm*[TIAB] OR self-harm*[TIAB] OR selfinjur*[TIAB] OR self-injur ${ }^{*}[T I A B]$ OR selfmutilat ${ }^{\star}[T I A B]$ OR self-mutilat ${ }^{\star}[T I A B]$ OR suicid*[TIAB] OR parasuicid*[TIAB) OR (suicide[TIAB] OR suicidal ideation [TIAB] OR attempted suicide[TIAB]) OR (drug overdose[TIAB] OR self?poisoning[TIAB]) OR (self-injurious behavio?r[TIAB] OR self?mutilation[TIAB] OR automutilation[TIAB] OR suicidal behavio?r[TIAB] OR self?destructive behavio?r[TIAB] OR self?immolation[TIAB])) OR (cutt* $[T I A B]$ OR head?bang[TIAB] OR overdose[TIAB] OR self?immolat*[TIAB] OR self?inflict* $\left.\left.\left.{ }^{\star}[T I A B]\right)\right)\right)$ AND ((coronavirus disease?19[TIAB] OR sars?cov?2[TIAB] OR mers?cov[TIAB]) OR (19?ncov[TIAB] OR 2019?ncov[TIAB] OR n? cov[TIAB]) \\ OR ("severe acute respiratory syndrome coronavirus 2" [Supplementary Concept] OR "COVID-19" [Supplementary Concept] OR COVID-19 [tw] OR coronavirus [tw] OR nCoV[TIAB] OR HCoV[TIAB] OR ((virus* [Title] OR coronavirus[Title] OR nCoV[Title] OR infectious[Title] OR HCoV[Title] OR novel[Title])AND (Wuhan[Title] OR China[Title] OR Chinese[Title] OR 2019[Title] OR 19[Title] OR COVID*[Title] OR \\ SARS-Cov-2[Title] OR NCP ${ }^{\star}[$ Title]) OR "Coronavirus"[MeSH]))))}

A two-stage screening process will be undertaken to identify studies meeting the eligibility criteria. First, two authors (either CO or EE) will assess citations from the searches and identify potentially relevant titles and abstracts. Second, either
DG, AJ or RW will assess the full texts of potentially eligible studies to identify studies to be included in the review. This process was managed via a custom-built online platform (Shiny web app, supported by a MongoDB database). The platform also allows for data extraction via a built-in form (extended data (John et al., 2020)). At the time of writing we are processing new records virtually every working day. Regularity of this task may reduce over time though it will be done at least monthly. We have the resources to display the current state of the review and screening process publicly on a regular basis.

\section{Data collection and assessment of risk of bias}

One author (DG, AJ or RW) will extract data from each included study using a piloted data extraction form (extended data (John et al., 2020)), and the extracted data will be checked by one other author. Disagreements will be resolved in the first instance through discussion, and where this fails, by referral to a third reviewer (KH or $\mathrm{NK})$. Irrespective of study design, data source and outcome measure examined, the following basic data will be extracted: citation; study aims and objectives; country/ setting; characteristics of participants; methods; outcome measures (related to suicidal behaviour and COVID-19); key findings; strengths and limitations; reviewer's notes. For articles where causal inferences are made i.e. randomized or non-randomized studies examining the effects of interventions or aetiological epidemiological studies of the effects of exposures, we will use a suitable version of the ROBINS-I or ROBINS-E tool to assess risk of bias as appropriate based on the research question and study design (Morgan et al., 2017; Sterne et al., 2016).

\section{Data synthesis}

We will synthesise studies according to themes based on research questions, using tables and narrative. Results will be synthesised separately for studies in the general population, in health and social care staff and other at-risk occupations, and in vulnerable populations (e.g. people of older age or those with underlying conditions that predispose them to becoming severely ill or dying after contracting Covid-19). Where multiple studies address the same research questions, we will assess whether meta-analysis is appropriate and conduct it where suitable following standard guidance available in the Cochrane Handbook (Deeks et al., 2019).

Living mode of the review, and transition to a static state We plan to maintain the review in living mode for at least 12 months, from publication of the protocol. We will undertake monthly screening and consider full updates considered at least every 3 months, if sufficient relevant evidence is published, although these are likely to happen monthly in the first instance. We will extend the living mode at 6-monthly intervals if evidence is still being published regularly. We anticipate an end to the living phase of the review at most 24 months after initiation, at which point we plan to publish the cumulated evidence in the form of a standard systematic review. Our decision to update the review will depend on the likely impact of the new evidence on the conclusions of the review. Impactful evidence may be (i) evidence that affects policy and/ or (ii) substantial, 
high-quality research studies (e.g. a randomised trial or population-based observational cohort study).

When a decision is made to move to from a living review to a static publication, two reviewers will independently assess $10 \%$ of all citations screened to determine whether any records were likely to have been missed, with further independent screening in the case of concerns. In addition, all extracted data will be checked against each relevant paper by an additional reviewer.

\section{Justification for a living method}

In the context of the COVID-19 pandemic there is a high level of uncertainty relating to its impact on suicide rates and how best to mitigate such effects and there is likely to be a rapidly emerging research evidence base to address this (Gunnell et al., 2020; Holmes et al., 2020; Reger et al., 2020). It is therefore important that the best available knowledge is made rapidly available to policymakers, public health specialists and clinicians to inform their practice.

\section{Dissemination of information}

This living review, along with further updates, will be published via F1000Research. This review was registered on PROSPERO, with ID CRD42020183326. All further data are publicly available via our Harvard Dataverse repository including all results of the continuous evidence surveillance and screening. Findings from the review will be widely disseminated through conference presentations, policy briefings, peer-reviewed publications, a project website, and traditional and social media outlets. Many of the co-authors are members of the International Covid-19 suicide prevention research collaboration (IPCSPRC), we will also use this network and its partnership with the International Association for Suicide Prevention to disseminate findings.
Study status

We are currently searching and screening on a daily basis.

\section{Data availability}

Underlying data

No data are associated with this article.

\section{Extended data}

Harvard Dataverse: Underlying data for: The impact of the Covid-19 pandemic on suicidal behaviour: a living systematic review protocol. https://doi.org/10.7910/DVN/9JYHLS (John et al., 2020)

This project contains the following extended data:

- Search.docx (additional information about the searches, including full search strategies)

- data_extraction_form.docx (Data extraction sheet/study report)

- 1sr_fig1.png (Figure 1 from protocol)

- PROSPERO_registration.pdf (Prospero registration)

\section{Reporting guidelines}

Harvard Dataverse: PRISMA-P checklist for 'The impact of the COVID-19 pandemic on self-harm and suicidal behaviour: protocol for a living systematic review' https://doi.org/10.7910/ DVN/9JYHLS (John et al., 2020)

\section{Software availability}

The development version of the software for automated searching is available from Github: https://github.com/mcguinlu/ COVID_suicide_living.

Archived source code at time of publication: http://doi.org/ 10.5281/zenodo.3871366 (McGuinness \& Schmidt, 2020)

License: MIT
Akl EA, Meerpohl JJ, Elliott J, et al:: Living systematic reviews: 4. Living guideline recommendations. J Clin Epidemiol. 2017; 91: 47-53. PubMed Abstract | Publisher Full Text

Chang SS, Stuckler D, Yip P, et al.: Impact of $\mathbf{2 0 0 8}$ global economic crisis on suicide: time trend study in 54 countries. BMJ. 2013; 347: f5239. PubMed Abstract | Publisher Full Text | Free Full Text

Cheung Y, Chau PH, Yip PSF: A revisit on older adults suicides and Severe Acute Respiratory Syndrome (SARS) epidemic in Hong Kong. Int J Geriatr Psychiatry. 2008; 23(12): 1231-1238. PubMed Abstract | Publisher Full Text

Deeks JJ, Higgins JP, Altman DG, et al:: Chapter 10: Analysing data and undertaking meta-analyses. Cochrane handbook for systematic reviews of interventions. 2nd ed. John Wiley \& Sons. 2019.

Reference Source

Elliott JH, Synnot A, Turner T, et al.: Living systematic review: 1. Introductionthe why, what, when, and how. J Clin Epidemiol. 2017; 91: 23-30. PubMed Abstract | Publisher Full Text

Elliott $\mathrm{JH}$, Turner T, Clavisi O, et al:: Living systematic reviews: an emerging opportunity to narrow the evidence-practice gap. PLoS Med. 2014; 11(2): e1001603.

PubMed Abstract | Publisher Full Text | Free Full Text
Gunnell D, Appleby L, Arensman E, et al:: Suicide risk and prevention during the COVID-19 pandemic. Lancet Psychiatry. 2020; 7(6): 468-471.

PubMed Abstract | Publisher Full Text | Free Full Text

Holmes EA, O'Connor RC, Perry VH, et al:: Multidisciplinary research priorities for the COVID-19 pandemic: a call for action for mental health science. Lancet Psychiatry. 2020; 7(6): 547-560.

PubMed Abstract | Publisher Full Text | Free Full Text

John J, Catherine Eyles E, McGuinness LA, et al.: Underlying data for: The impact of the Covid-19 pandemic on suicidal behaviour: a living systematic review protocol. Harvard Dataverse, V2. 2020.

http://www.doi.org/10.7910/DVN/9JYHLS

Johnson NPAS, Mueller J: Updating the accounts: global mortality of the 1918-1920" Spanish" influenza pandemic. Bull Hist Med. 2002; 76(1): 105-115. PubMed Abstract | Publisher Full Text

Kisely S, Warren N, McMahon L, et al:: Occurrence, prevention, and management of the psychological effects of emerging virus outbreaks on healthcare workers: rapid review and meta-analysis. BMJ. 2020; 369: m1642. PubMed Abstract | Publisher Full Text | Free Full Text

McGuinness L, Schmidt L: mcguinlu/COVID suicide_living: Initial Release (Version v1.0.0). Zenodo. 2020.

http://www.doi.org/10.5281/zenodo.3871366 
Moher D, Shamseer L, Clarke M, et al:: Preferred reporting items for systematic review and meta-analysis protocols (PRISMA-P) 2015 statement. Syst Rev. 2015; 4(1): 1.

PubMed Abstract | Publisher Full Text | Free Full Text

Morgan R, Sterne JA, Higgins JP, et al.: A new instrument to assess Risk of Bias in Non-randomised Studies of Exposures (ROBINS-E): Application to studies of environmental exposure Global Evidence Summit. Cape Town. 2017 Reference Source

Reger MA, Stanley IH, Joiner TE: Suicide Mortality and Coronavirus Disease 2019-A Perfect Storm? JAMA Psychiatry. 2020.

PubMed Abstract | Publisher Full Text

Sterne JA, Hernán MA, Reeves BC, et al.: ROBINS-I: a tool for assessing risk of bias in non-randomised studies of interventions. BMJ. 2016; 355: i4919. PubMed Abstract | Publisher Full Text | Free Full Text

Stuckler D, Basu S, Suhrcke M, et al.: The public health effect of economic crises and alternative policy responses in Europe: an empirical analysis. Lancet. 2009; 374(9686): 315-323.

PubMed Abstract | Publisher Full Text

Turecki G, Brent DA, Gunnell D, et al:: Suicide and suicide risk. Nat Rev Dis Primers. 2019; 5(1): 74

PubMed Abstract | Publisher Full Text

Wasserman IM: The impact of epidemic, war prohibition and media on suicide: United States, 1910-1920. Suicide Life Threat Behav. 1992; 22(2): 240-254.

PubMed Abstract 


\section{Open Peer Review}

\section{Current Peer Review Status:}

\section{Version 1}

Reviewer Report 29 July 2020

https://doi.org/10.5256/f1000research.26781.r65519

(C) 2020 Duncan E. This is an open access peer review report distributed under the terms of the Creative Commons Attribution License, which permits unrestricted use, distribution, and reproduction in any medium, provided the original work is properly cited.

\section{Edward A. S. Duncan}

Nursing, Midwifery and Allied Health Professional Research Unit, University of Stirling, Stirling, UK

This paper presents a protocol for a living systematic review on the impact of COVID-19 pandemic on self-harm and suicidal behaviour. This is an important and timely review.

Suicidal and self-harm behaviours have increased during previous epidemics and pandemics and there are good theoretical reasons to believe that a similar patter will occur during the COVID-19 pandemic. The review aims to rapidly provide real-time evidence of the impact of COVID-19 pandemic on suicide deaths, suicidal behaviours, self-harm, and suicidal thoughts, and to assess the effectiveness of strategies that aim to reduce these.

The review is a mixed-method in design and will include both quantitative and qualitative (except single case studies in either method). The protocol is well described and presented in line with PRISMA guidelines.

PsychInfo is not included as one of the listed search engines, which appears to be an omission. The link to medRxiv is broken.

Factors for the authors to consider are as follows. How the authors will assess bias in quantitative research is clearly described. However, it is unclear how the authors will review the quality of qualitative studies. It is also unclear how the qualitative data will be synthesised with the quantitative data and inform the review of effectiveness study. The living systematic-review design lends itself to a greater integration of both quantitative and qualitative data as they are published.

The transition of the review to a static state and the dissemination strategy is well described.

Is the rationale for, and objectives of, the study clearly described? Yes

Is the study design appropriate for the research question? 
Yes

Are sufficient details of the methods provided to allow replication by others?

Yes

Are the datasets clearly presented in a useable and accessible format?

Not applicable

Competing Interests: No competing interests were disclosed.

Reviewer Expertise: Mental Health and pre-hospital emergency care research. Currently undertaking 3 COVID-19 related studies, including two in mental health contexts.

I confirm that I have read this submission and believe that I have an appropriate level of expertise to confirm that it is of an acceptable scientific standard.

Reviewer Report 02 July 2020

https://doi.org/10.5256/f1000research.26781.r65517

(c) 2020 Turner T. This is an open access peer review report distributed under the terms of the Creative Commons Attribution License, which permits unrestricted use, distribution, and reproduction in any medium, provided the original work is properly cited.

\section{Tari Turner}

Monash University, Melbourne, Australia

This is a clear and well written protocol for a living systematic review of evidence on the impact of the COVID-19 pandemic on self-harm and suicidal behaviour. This is an important topic, a living systematic review approach is clearly useful for this topic at this time, and the methods described are also appropriate.

I note that the review questions are complex and this will both make the review more useful, but also more challenging to do and to describe.

Is there any plan to review the search strategy to ensure the terms used remain appropriate? Will non-English studies be included? Have searches of trials registers been conducted to determine the likely workload over coming months?

I would encourage the authors to provide more clarity on the approach and criteria they will use to make decisions about:

changing the frequency of screening

whether there is "sufficient relevant evidence" to justify an update.

Ideally enough detail should be provided on these to allow another team to make the same decisions, using the same criteria; and I don't think this is true of the descriptions as they currently 
stand.

For example, some other living review teams have committed to updating their reviews when new evidence is likely to have a clinically meaningful impact on the size of the effect, or change the direction or degree of certainty (GRADE) of the effect for key outcomes. I recognise that the complexity of the review questions and types of evidence being included in this review may make this challenging, but additional explanation would be helpful, and avoid potential future perceptions of bias based on these decisions. (These decisions may also require that some elements of data extraction and ROB assessment are taken before a decision to update is undertaken, and this should be considered for workflows.)

Also there is some confusion between the Figure and the text, with the figure appearing to suggest that update decisions are made 3 monthly, while the text suggests these decisions may initially be more frequent (monthly?); and the figure suggesting that screening is conducted daily (presumably 5/7?) but there is some ambiguity about commitment to this in the text.

I recommend review of the article by a person with clinical expertise in the area, as I do not have these skills, and I look forward to reading the review.

Is the rationale for, and objectives of, the study clearly described?

Yes

Is the study design appropriate for the research question?

Yes

Are sufficient details of the methods provided to allow replication by others? Partly

Are the datasets clearly presented in a useable and accessible format? Not applicable

Competing Interests: No competing interests were disclosed.

Reviewer Expertise: Living evidence synthesis methods.

I confirm that I have read this submission and believe that I have an appropriate level of expertise to confirm that it is of an acceptable scientific standard, however I have significant reservations, as outlined above. 
The benefits of publishing with F1000Research:

- Your article is published within days, with no editorial bias

- You can publish traditional articles, null/negative results, case reports, data notes and more

- The peer review process is transparent and collaborative

- Your article is indexed in PubMed after passing peer review

- Dedicated customer support at every stage

For pre-submission enquiries, contact research@f1000.com 